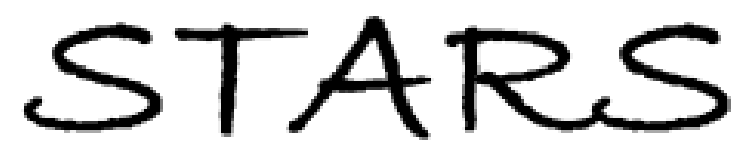

University of Central Florida

STARS

$1-1-2000$

\title{
Relative electrical resistivities and poling of nonlinear optical polymeric waveguides
}

\author{
Tomáš Pliška \\ University of Central Florida \\ Joachim Meier \\ University of Central Florida \\ Arne Eckau \\ University of Central Florida \\ Vincent Ricci \\ University of Central Florida
}

Anne-Claire Le Duff

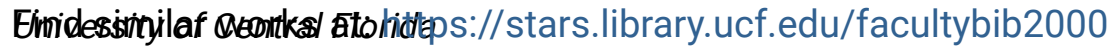

University of Central Florida Libraries http://library.ucf.edu

See next page for additional authors

This Article is brought to you for free and open access by the Faculty Bibliography at STARS. It has been accepted for inclusion in Faculty Bibliography 2000s by an authorized administrator of STARS. For more information, please

contactSTARS@ucf.edu.

\section{Recommended Citation}

Pliška, Tomáš; Meier, Joachim; Eckau, Arne; Ricci, Vincent; Duff, Anne-Claire Le; Canva, Michael; Stegeman, George I.; Raymond, Paul; Kajzar, Francois; and Chan, Kwok Pong, "Relative electrical resistivities and poling of nonlinear optical polymeric waveguides" (2000). Faculty Bibliography 2000 s.

2744.

https://stars.library.ucf.edu/facultybib2000/2744

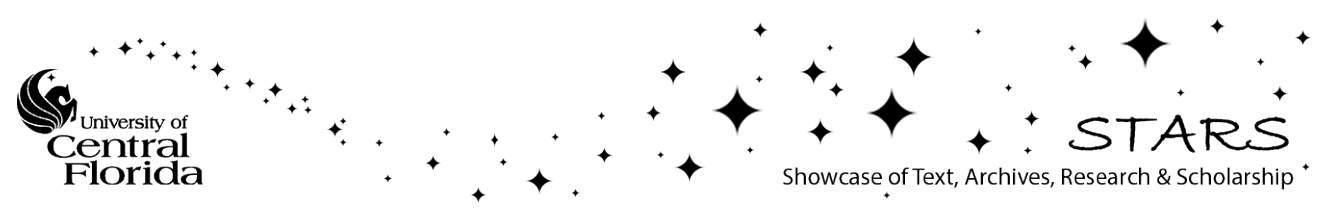




\section{Authors}

Tomáš Pliška, Joachim Meier, Arne Eckau, Vincent Ricci, Anne-Claire Le Duff, Michael Canva, George I.

Stegeman, Paul Raymond, Francois Kajzar, and Kwok Pong Chan 


\section{Relative electrical resistivities and poling of nonlinear optical polymeric waveguides}

Cite as: Appl. Phys. Lett. 76, 265 (2000); https://doi.org/10.1063/1.125742

Submitted: 01 October 1999 . Accepted: 17 November 1999 . Published Online: 10 January 2000

Tomáš Pliška, Joachim Meier, Arne Eckau, Vincent Ricci, Anne-Claire Le Duff, Michael Canva, George I. Stegeman, Paul Raymond, François Kajzar, and Kwok Pong Chan 


\title{
Relative electrical resistivities and poling of nonlinear optical polymeric waveguides
}

\author{
Tomáš Pliška, ${ }^{\text {a) }}$ Joachim Meier, Arne Eckau, ${ }^{\text {b) }}$ Vincent Ricci, Anne-Claire Le Duff, ${ }^{c)}$ \\ Michael Canva, ${ }^{\mathrm{c})}$ and George I. Stegeman \\ School of Optics/Center for Research and Education in Optics and Lasers (CREOL), \\ University of Central Florida, P.O. Box 162 700, 4000 Central Florida Boulevard, Orlando, \\ Florida 32816-2700 \\ Paul Raymond and François Kajzar \\ Commissariat à l'Énergie Atomique (CEA), Centre d'Études de Saclay, 91191 Gif sur Yvette Cedex, France \\ Kwok Pong Chan \\ Molecular OptoElectronics Corporation (MOEC), 877 25th Street, Watervliet, New York 12189-1903
}

(Received 1 October 1999; accepted for publication 17 November 1999)

A method based on Maker fringe measurements of nonlinear optical coefficients has been used to determine the relative dc electrical resistivities of a series of linear and nonlinear optical polymers. The method can be used to identify low resistivity linear cladding materials for optimized electric field poling of nonlinear optical polymeric waveguides. As an example of the application of the technique we have studied the resistivity of poly(methyl methacrylate)-polystyrene (PMMA-PS) copolymers with varying content of the two components. The resistivity of PMMA was found to be one order of magnitude lower than that of PS. (C) 2000 American Institute of Physics.

[S0003-6951(00)01603-X]

Organic materials, both in single crystal and poled polymer form, can exhibit large optical nonlinearities and are thus attractive for many applications. ${ }^{1-3}$ Poled polymers have shown great potential for high frequency electro-optic modulators because of their low dielectric dispersion and fast response. ${ }^{4}$ Other, more recently evolving applications include frequency conversion and cascading at telecommunication wavelengths. ${ }^{5}$ Guided-wave configurations are particularly suitable for nonlinear optics because of the high optical intensity that can be maintained over a long interaction length in the waveguide. ${ }^{6}$

The basic structure underlying most nonlinear polymeric waveguides is shown schematically in Fig. 1(a). A high index core layer containing the nonlinear optical chromophores is sandwiched between two low index linear cladding layers. Depending on the application, more layers can be included in the structure. Typical layer thicknesses are on the order of a few hundred nanometers to several micrometers. A secondorder nonlinearity in the core layer is induced by applying a dc electric field which partially aligns the dipole moments of the chromophores. This process is referred to as electric field poling. ${ }^{7}$ For the structure shown in Fig. 1(a) with the electrodes at the bottom and on top of the waveguide (parallel plate poling), the poling voltage is applied across all three layers and will divide according to their resistances. Thus, the effective poling field across the nonlinear core layer will depend on the thicknesses and relative resistivities of the

\footnotetext{
${ }^{a)}$ Present address: JDS Uniphase AG, Binzstrasse 17, 8045 Zürich, Switzerland; electronic mail: tomas.pliska@ch.jdsunph.com

${ }^{b}$ Permanent address: Institut für Schicht- und Ionentechnik ISI-2, Forschungszentrum Jülich, 52425 Jülich, Germany.

c)Permanent address: Laboratoire Charles Fabry, Institut d'Optique Théorique et Appliquée (IOTA), Université d'Orsay/Paris-Sud, 91403 Orsay Cedex, France.
}

materials used in the structure. High resistivity cladding layers will severely reduce the poling field in the core nonlinear layer resulting in a reduction or complete loss of the optical nonlinearity.

Typical specific ohmic resistivities of polymers range from $10^{11}$ to $10^{24} \Omega \mathrm{m}$. The task of measuring resistivities of thin polymer films is not trivial, and values quoted in the literature for a given material usually span several orders of magnitude, depending on, e.g., sample preparation, degree of polymerization, layer quality, purity, etc. Simple currentvoltage $(I-V)$ measurements do not provide the required information, since the $I-V$ characteristic is often determined by interfacial effects between the polymer and the electrodes rather than by the bulk properties of the polymer film itself. ${ }^{8}$ In this work we have used a nonlinear optical method to determine relative poling efficiencies.

The sample structure for our measurements is shown in Fig. 1(b). It is composed of two polymer layers, one of which consists of a nonlinear optical polymer (thickness $t_{1}$,

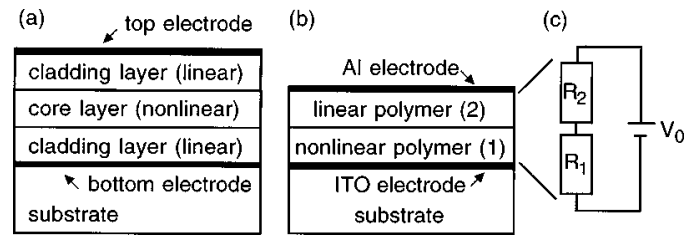

FIG. 1. (a) Basic structure of a nonlinear optical polymeric waveguide with three polymer layers (lower cladding, core, upper cladding) spin coated on a substrate and sandwiched between two poling electrodes. (b) Structure of the samples used in this work to measure relative resistivities consisting of one layer of nonlinear-optical polymer (1) and a linear cladding polymer (2) between an indium-tin-oxide (ITO) and an aluminum electrode. (c) Equivalent electrical circuit for dc electric poling of the structure shown in (b), with $R_{1}$ and $R_{2}$ denoting the resistance of the nonlinear and linear layer, respectively, and $V_{0}$ the applied poling voltage. 
specific resistivity $\rho_{1}$ ) while the other one is a linear cladding polymer (thickness $t_{2}$, specific resistivity $\rho_{2}$ ). A dc voltage $V_{0}$ is then applied across the two-layer structure. Neglecting capacitive effects (which is valid for dc fields and sufficiently long relaxation times) and contact resistances (which are assumed to be much smaller than the material resistivities), the electrical circuit can be simply represented by two resistances in series, with $R_{1}$ denoting the resistance of the nonlinear layer and $R_{2}$ the resistance of the cladding layer [Fig. 1(c)]. The nonlinearity induced by the poling process is a direct measure of the effective poling field $E_{1}$ across the nonlinear layer during poling since thermodynamic considerations predict a linear dependence of the nonlinearity on the poling field in the low field limit. ${ }^{9}$

For the analysis, we define the total thickness $t_{0} \equiv t_{1}$ $+t_{2}$ and a nominal (average) electric field across the two layers $E_{0} \equiv V_{0} / t_{0}$. A straightforward calculation using Ohm's law leads to the following expression for the external field across the nonlinear layer $E_{1}$ :

$$
\frac{E_{1}}{E_{0}}=\frac{1+\frac{t_{2}}{t_{1}}}{1+\frac{\rho_{2}}{\rho_{1}} \frac{t_{2}}{t_{1}}} .
$$

Notice that only relative variables appear in Eq. (1).

Samples were prepared by spin coating $0.5-5 \mu \mathrm{m}$ thick polymer layers on glass substrates coated with indium-tinoxide (ITO) as the bottom transparent electrode. The polymers were dried in an oven for several hours. The layer thicknesses were measured with an alpha step 200 profilometer (accuracy $\pm 1 \%$ ). A $150 \mathrm{~nm}$ thick aluminum layer was deposited on top as the upper electrode. Poling was done by heating the sample to an elevated temperature and then applying a voltage corresponding to a nominal field $E_{0}$ of 50$100 \mathrm{~V} \mu \mathrm{m}^{-1}$. After cooling down the sample the voltage was turned off, and the top electrode was removed using an $\mathrm{Al}$ etch solution. Maker fringe measurements at a wavelength of $1.58 \mu \mathrm{m}$ were performed to determine the nonlinearity. The configuration of our setup allowed us to measure the nonlinear-optical coefficient $d_{31}$ which will be denoted as $d$ in the following (accuracy of Maker fringe measurement $\pm 15 \%$, minimum resolution $0.1 \mathrm{pm} \mathrm{V}^{-1}$ ).

We used two different nonlinear optical polymers, namely 4 -[ $N$-ethyl- $N$-(2-hydroxyethyl)]amino- 4 '-nitroazobenzene) (disperse red 1, DR1; glass transition temperature $T_{g}=131^{\circ} \mathrm{C}$, number density of chromophores $N=0.5$ $\times 10^{27} \mathrm{~m}^{-3}$, poling temperature $T_{\mathrm{pol}}=120^{\circ} \mathrm{C}$ ), and 4-diethylamino-1-nitrobenzyl (DANB; $T_{g}=100^{\circ} \mathrm{C}, N=2.6$ $\times 10^{27} \mathrm{~m}^{-3}, T_{\mathrm{pol}}=100^{\circ} \mathrm{C}$ ), both covalently attached to a poly(methyl methacrylate) backbone forming a side chain polymer. In order to calibrate the nonlinearity versus poling field curve, we first measured the nonlinear coefficients $d$ using single layer samples of each nonlinear polymer without a cladding layer on top $\left(t_{2}=0\right)$. The corresponding measurements are shown in Figs. 2(a) and 2(b) with the lines indicating linear fits. For the slopes we obtain values of $(1.57 \pm 0.11) \times 10^{-20} \mathrm{~m}^{2} \mathrm{~V}^{-2}$ for DANB and $(1.73 \pm 0.15)$ $\times 10^{-20} \mathrm{~m}^{2} \mathrm{~V}^{-2}$ for DR1. Using these relations we can then deduce the effective field in the nonlinear layer of the poled composite two layer (linear-nonlinear) samples. (a)

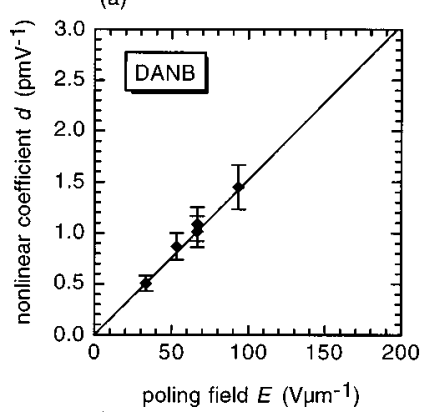

(b)

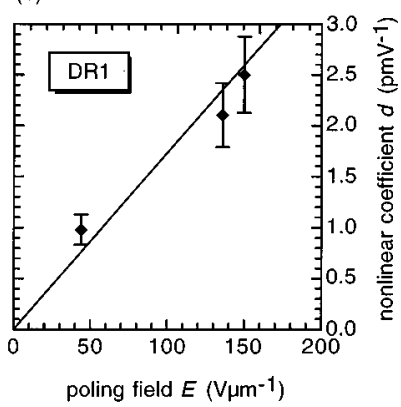

FIG. 2. Measured nonlinear-optical coefficient $d\left(=d_{31}\right)$ as a function of the applied external poling field for single-layer samples of the two nonlinear polymers DANB (a) and DR1 (b) used in this work. The lines indicate linear fits to the data.

Several combinations of these two nonlinear polymers with linear cladding polymers were then investigated for their nonlinearity. We used poly(methyl methacrylate) (PMMA, $T_{g}=105^{\circ} \mathrm{C}$ ), polystyrene (PS, $T_{g}=98^{\circ} \mathrm{C}$ ), polycarbonate (PC, $\left.T_{g}=150^{\circ} \mathrm{C}\right)$, and copolymers of PMMA and PS as cladding materials. In Table I we have listed the measured relative resistivities $\rho_{2} / \rho_{1}$ at the previously mentioned poling temperatures of 100 and $120^{\circ} \mathrm{C}$. For some polymer combinations the measurement was performed on several samples, and the error was weighted accordingly. Remarkable differences in the resistivities of the different linear polymers can be noted, e.g., PS having a resistivity about one order of magnitude larger than PMMA. Our measurements are in accordance with the trends for the volume resistivities of these polymers found in the literature. ${ }^{10}$ No systematic dependence of the resistivity of the cladding polymers on their glass transition temperatures was apparent in our studies.

The thickness dependence of the effective poling field was investigated using the DR1/PMMA and DR1/PS structures. In Fig. 3 we plotted the relative effective poling field $E_{1} / E_{0}$ as a function of the thickness ratio $t_{2} / t_{1}$ (accuracy $\pm 20 \%)$. The lines indicate fits according to Eq. (1) with $\rho_{2} / \rho_{1}$ as a free fit parameter, yielding values of $0.6 \pm 0.3$ and $4.7 \pm 0.9$ for the DR1/PMMA and DR1/PS samples, respec-

TABLE I. Relative resistivities $\rho_{2} / \rho_{1}$ of linear and nonlinear optical polymers measured with samples shown schematically in Fig. 1(b). The linear polymers used were polycarbonate (PC), poly(methyl methacrylate) (PMMA), polystyrene (PS), and copolymers of PMMA and PS, while the nonlinear optical chromophores were 4-[N-ethyl-N-(2-hydroxyethyl)] amino-4'-nitroazobenzene) (disperse red 1, DR1) and 4-diethylamino-1nitrobenzyl (DANB), both attached to a PMMA backbone. The poling temperature was $100^{\circ} \mathrm{C}$ for the DANB and $120^{\circ} \mathrm{C}$ for the DR1 samples. The numbers in brackets indicate the number of samples used for the measurement.

\begin{tabular}{lc}
\hline \hline Linear polymer/nonlinear polymer & Relative resistivity $\rho_{2} / \rho_{1}$ \\
\hline PC/DANB & $0.9 \pm 0.3(1)$ \\
PMMA/DANB & $2.6 \pm 0.4(3)$ \\
$(\mathrm{PMMA})_{0.75}-(\mathrm{PS})_{0.25} / \mathrm{DANB}$ & $3.6 \pm 0.9(2)$ \\
$\left.(\mathrm{PMMA})_{0.5}-\mathrm{PS}\right)_{0.5} / \mathrm{DANB}$ & $4.6 \pm 2.6(1)$ \\
$(\mathrm{PMMA})_{0.25}-(\mathrm{PS})_{0.75} / \mathrm{DANB}$ & $8.3 \pm 5.7(2)$ \\
PS/DANB & $12.1 \pm 4.3(2)$ \\
PMMA/DR1 & $0.6 \pm 0.3(4)$ \\
$(\mathrm{PMMA})_{0.5}-(\mathrm{PS})_{0.5} / \mathrm{DR} 1$ & $1.2 \pm 0.4(1)$ \\
PS/DR1 & $4.7 \pm 0.9(4)$ \\
\hline \hline
\end{tabular}




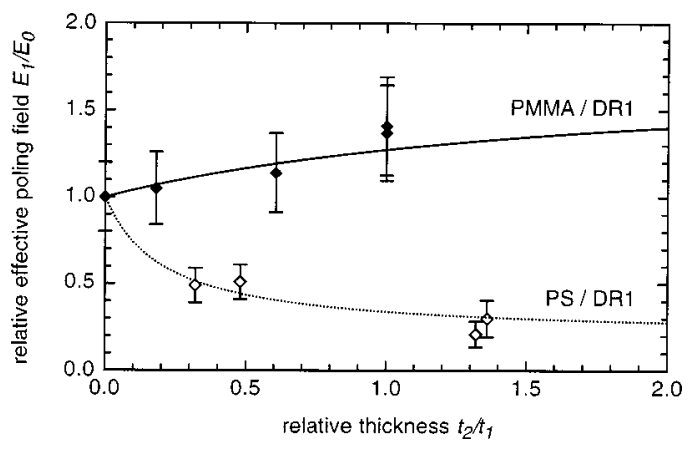

FIG. 3. Relative effective poling field $E_{1} / E_{0}$ across the nonlinear polymer for DR1/PMMA and DR1/PS samples as a function of the relative thickness of the two layers. The field $E_{1}$ was deduced from Maker fringe measurements of the nonlinear-optical coefficient of the DR1 layer. The lines indicate fits according to Eq. (1) with the relative resistivity $\rho_{2} / \rho_{1}$ as a free parameter yielding $\rho_{2} / \rho_{1}=0.6 \pm 0.3$ for PMMA/DR1 and $4.7 \pm 0.9$ for PS/ DR1.

tively. The ratio $E_{1} / E_{0}$ is equal to the relative nonlinearity $d / d_{0}$ where $d_{0}$ is the nonlinear coefficient of a single layer poled at field $E_{0}$. The results shown in Table I and Fig. 3 clearly illustrate the importance of optimizing the relative resistivities for poling. Use of high resistivity cladding polymers, in this example PS, can reduce the nonlinearity to less than $10 \%$ of the nonlinearity expected for the field $E_{0}$.

Because of the large difference between the resistivities of PMMA and PS we performed a measurement where copolymers of PMMA and PS with varying ratio of the two components were used as cladding layers. The resistivity of the PMMA-PS copolymers relative to DANB and DR1, respectively, is plotted in Fig. 4 as a function of the PMMA mole fraction. The resistivity decreases with increasing PMMA content, as expected from the measurement shown in Fig. 3. The large error bars for the DANB samples are due to the small nonlinear coefficients measured for this polymer. This measurement illustrates that the electrical properties can be tuned by means of copolymerization, keeping in mind, however, that other properties like the linear refractive index or glass transition temperature change as well.

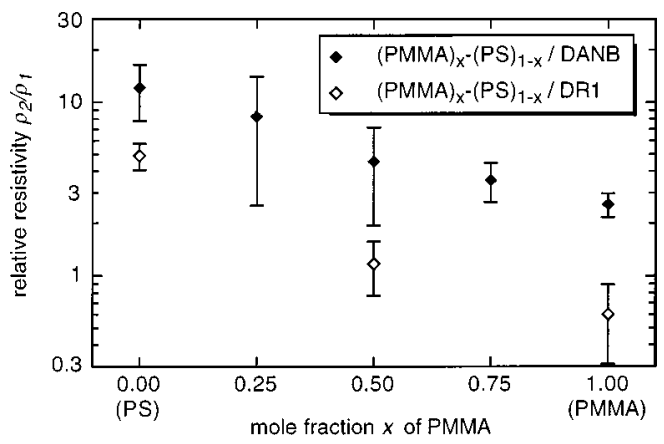

FIG. 4. Relative resistivity $\rho_{2} / \rho_{1}$ of (PMMA $)_{x}-(\mathrm{PS})_{1-x}$ copolymers and DANB (upper data points) and DR1 (lower data points), respectively, as a function of the PMMA mole fraction $x$ in the copolymer. The values for pure PMMA and PS are given at $x=1$ and $x=0$, respectively. The poling temperature was $100^{\circ} \mathrm{C}$ for the DANB and $120^{\circ} \mathrm{C}$ for the DR1 samples.
In conclusion we have used a technique based on Maker fringe measurements of the nonlinearity to directly evaluate the poling efficiency in stacks of nonlinear and linear polymer layers. This method is suitable for identifying low resistivity cladding materials for improved poling efficiency. Using a nonlinear (linear) polymer material with a known absolute specific resistivity as a standard, it would also allow the determination of the absolute resistivities of linear (or nonlinear) polymers. Error analysis shows that the main uncertainty in the measured relative resistivity $\rho_{2} / \rho_{1}$ is due to the uncertainty in the measured nonlinear coefficient (Maker fringe measurement) and the fitted slope of the nonlinearityfield dependence (Fig. 2). The experimental error decreases with increasing nonlinear coefficient and increasing layer thicknesses. The relative error of the quantity $\rho_{2} / \rho_{1}$ from a single measurement can vary between $25 \%$ and $100 \%$, and thus several independent measurements may be required to improve the accuracy. The precision of the technique is further reduced if the relative resistivities differ by more than an order of magnitude. Moreover, the method can be used only if the contact resistances are negligible which requires the use of electrode materials with low work functions. However, within these limits, this method is a useful tool for studying the dc electrical properties of linear and nonlinearoptical polymers with respect to their implementation in waveguide devices. In particular, it probes directly the effective poling field without measurement of the absolute resistivity. As an example of the application of this technique we have investigated the electrical properties of PMMA-PS copolymers with varying molar ratio. The method could be further used to study for example the effect of varying chromophore loading on the resistivity of the nonlinear polymer or to evaluate the optimized poling temperature for a given material system.

This research was supported by AFSOR through JPL Contract No. 961393 and by BMDO STTR Grant No. F29601-98-C-0205. T.P. wishes to acknowledge financial support by Swiss National Science Foundation.

${ }^{1}$ Molecular Nonlinear Optics, edited by J. Zyss (Academic, San Diego, CA, 1994).

${ }^{2}$ Ch. Bosshard, K. Sutter, Ph. Prêtre, J. Hulliger, M. Flörsheimer, Ph. Kaatz, and P. Günter, Organic Nonlinear Optical Materials (Gordon and Breach Publishers, Basel, 1995).

${ }^{3}$ Nonlinear Optics of Organic Molecules and Polymers, edited by H. S. Nalwa and S. Miyata (CRC, Boca Raton, FL, 1997).

${ }^{4}$ D. Chen, H. R. Fetterman, A. Chen, W. H. Steier, L. R. Dalton, W. Wang, and S. Yongqiang, Appl. Phys. Lett. 70, 3335 (1997).

${ }^{5}$ M. Jäger, G. I. Stegeman, S. Yilmaz, W. Wirges, W. Brinker, S. BauerGogonea, S. Bauer, M. Ahlheim, M. Stählin, B. Zysset, F. Lehr, M. Diemeer, and M. C. Flipse, J. Opt. Soc. Am. B 15, 781 (1998).

${ }^{6}$ G. I. Stegeman and R. H. Stolen, J. Opt. Soc. Am. B 6, 652 (1989).

${ }^{7}$ K. D. Singer, J. E. Sohn, and S. J. Lalama, Appl. Phys. Lett. 49, 248 (1986).

${ }^{8}$ R. Blum, M. Sprave, J. Sablotny, and M. Eich, J. Opt. Soc. Am. B 15, 318 (1998).

${ }^{9}$ K. D. Singer, M. G. Kuzyk, and J. E. Sohn, J. Opt. Soc. Am. B 4, 968 (1987).

${ }^{10}$ J. Brandrup, E. H. Immergut, and E. A. Grulke, Polymer Handbook, 4th ed. (Wiley, New York, 1999). 